\title{
Checklist of the genus Aparasphenodon Miranda-Ribeiro, 1920 (Anura: Hylidae): Distribution map, and new record from São Paulo state, Brazil
}

\author{
Antonio Mollo Neto ${ }^{1^{*}}$ and Mauro Teixeira Jr. ${ }^{2}$ \\ 1 Universidade Federal do ABC, Centro de Ciências Naturais e Humanas, Laboratório de Vertebrados. Av. dos Estados 5001, Bloco A, $6^{\circ}$ andar \\ DI624. CEP 09210-971. Santo André, SP, Brazil. \\ 2 Universidade de São Paulo, Instituto de Biociências, Departamento de Zoologia. Rua do Matão, Travessa 14, № 102. Caixa Postal 11.461. CEP \\ 05422-970. São Paulo, SP, Brazil. \\ * Corresponding author. E-mail: mollonetoa@gmail.com
}

ABstract: The distribution of the genus Aparasphenodon is mapped and we report the southernmost record of Aparasphenodon brunoi from São Paulo state, Brazil.

The genus Aparasphenodon (Casque-headed frogs) was originally described by Miranda-Ribeiro (1920) with A. brunoi as the type species. In the same publication the author also described Corythomantis apicalis from Espírito Santo (Brazil). Five years later, Lutz (1925) described $C$. adspersa, from Rio de Janeiro, Brazil. Both C. apicalis and C. adspersa were later synonymized with $A$. brunoi by Carvalho (1941). No type locality was originally provided by Miranda-Ribeiro (1920) for $A$. brunoi, but the matter was cleared later as the specimens were obtained from Rio de Janeiro (Miranda-Ribeiro 1955).

Five decades later the genus was still monotypic and Trueb (1970), after a morphological assessment of Aparasphenodon, placed Corythomantis venezolana, described by Mertens (1950), in the genus Aparasphenodon. This revealed a substantial disjunct distribution, as Aparasphenodon brunoi occurs in the Atlantic Forest of eastern Brazil and A. venezolanus in the Amazon forest of southern Venezuela.

After approximately two decades, a new species from a more southern part of the Atlantic Forest, Aparasphenodon bokermanni, was described (Pombal 1993). And a few years ago the last known species (A. arapapa) was described from the northernmost limit of Aparasphenodon range along the Atlantic Forest, in coastal Bahia (Pimenta et al. 2009).

The most conspicuous feature among Aparasphenodon species is the strongly co-ossified skin skull, especially in $A$. brunoi, a character that seems to be related to phragmotic behavior and may provide extra protection while hiding inside bromeliad tanks and bamboo holes (Teixeira et al. 2002; Mesquita et al. 2004).

Here we provide an updated distributional map of Aparasphenodon species compiled from the literature, online databases (ZUEC, MBML and CFBH collections were accessed through SpeciesLink web portal, vouchers not examined) (SpeciesLink 2011), and museum specimens from the Museu de Zoologia da Universidade de São
Paulo (MZUSP) (Tables 1-4). Additionally, we report the southernmost record of $A$. brunoi from São Paulo state, Brazil.

The distribution of the genus can be summarized as follows: Aparasphenodon venezolanus is found in the Amazon forest of southwestern Venezuela, eastern Colombia and northern Brazil, and is known only from a few localities (Table 1) along the Rio Negro basin; $A$. arapapa is known from a few localities only (Table 2), in the Atlantic Forest, between Paraguaçu and Jequitinhonha rivers, in Bahia state; A. bokermanni is also known only from a few localities (Table 3) in the subtropical Atlantic Forest of southern São Paulo and Santa Catarina states, and is expected to occur in the geographically intermediate state of Parana, although no individuals have been recorded there yet; $A$. brunoi is widely distributed over the Atlantic Forest, and is reported from several localities (Table 4) between northern coastal São Paulo state and Bahia (Figure 1).

Sazima and Cardoso (1980), and later Gioia and Souza-Lima (1988) recorded Aparasphenodon brunoi in the restingas of Lázaro beach, Ubatuba municipality, in São Paulo state. More than twenty years have passed and no other locality of $A$. brunoi in São Paulo state has been recorded.

In August 2009 we collected, a juvenile male of Aparasphenodon brunoi (snout-vent length $41.1 \mathrm{~mm}$ ) (Figure 2), which was found immobile inside an epiphytic bromeliad, $50 \mathrm{~cm}$ above the ground, at about 20:00 h, on the border of a small restinga forest fragment, no longer than $200 \mathrm{~m}$, in Caraguatatuba municipality, coastal São Paulo state $\left(23^{\circ} 39^{\prime} 26.6^{\prime \prime} \mathrm{S}, 45^{\circ} 25^{\prime} 56.62^{\prime \prime} \mathrm{W}\right.$, WGS 84, $5 \mathrm{~m}$ a.s.l.). Our record confirms that $A$. brunoi is still present in the area, and represents the second locality for the species in São Paulo, as well as the southernmost locality of its distributional range, extending it approximately $35 \mathrm{~km}$ southwestwards along the coast of São Paulo (Figure 3).

The specimen was euthanized using a lethal dose of 
anesthesics, fixed in $10 \%$ formalin, preserved in $70 \%$ alcohol (ICMBio permit number 14555-3), and deposited in the herpetological collection of Museu de Zoologia da Universidade de São Paulo, São Paulo (MZUSP 149809).

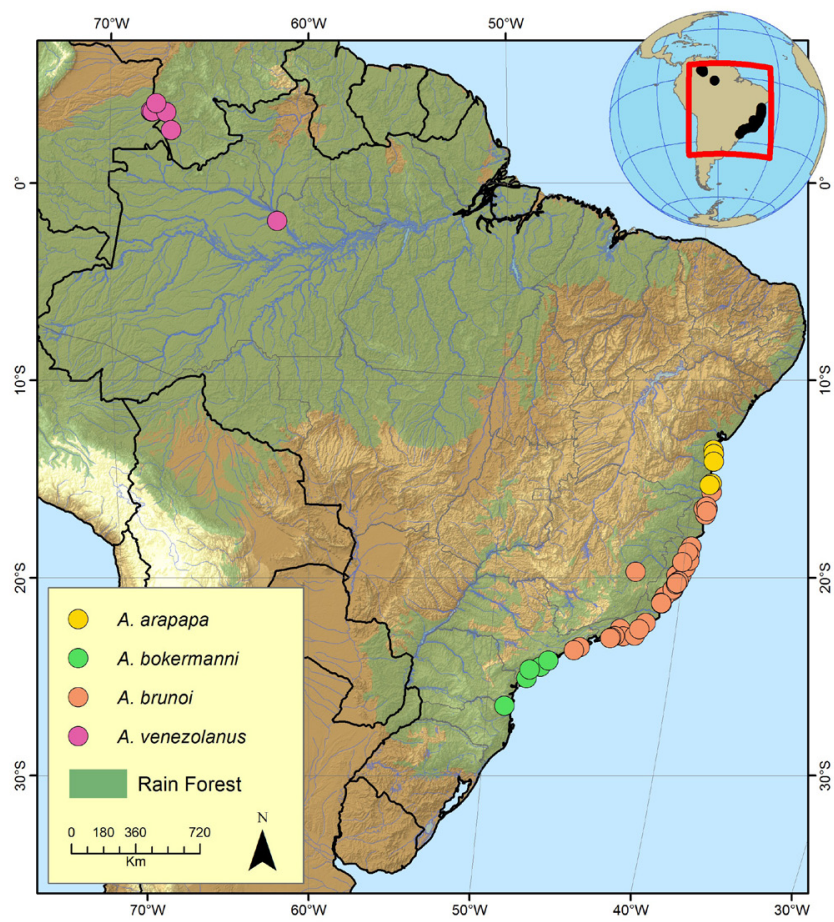

FIGURE 1. Geographic distribution map of Aparasphenodon species.
Although very common in coastal restingas and some inland forests in the northern part of its distribution, Aparasphenodon brunoi seems to be relatively rare at the southern end of its distribution, although ground and epiphytic bromeliads are very abundant in that area. This local rarity could be related to subtropical conditions, which cause lower temperatures along the coastal plain, thus working as a putative physiological barrier. Recently substantial populations of the collared lizard Tropidurus torquatus (Sena et al. 2008) and of an anole lizard (the authors, unpublished data) have been found on the northern São Paulo coast, where despite large previous sampling efforts they had never been recorded, (or only from a few specimens). This southern expansion was hypothesized to be related to global warming, allowing those lizards to disperse southwards and colonize new regions that they could not reach before (Sena et al. 2008).

It is difficult to evaluate whether the occurrence of Aparasphenodon brunoi south of Ubatuba is a recent expansion or the result of increased herpetofaunal sampling efforts. However habitat destruction is certainly shrinking the species distribution. The area in Caraguatatuba where we found the specimen is a small circular fragment of restinga forest, no more than $200 \mathrm{~m}$ long, and not connected with any larger continuous piece of forest. The collection locality is concealed within a highly populated region where most of the original habitat has been completely removed.

TABLE 1. Localities for Aparasphenodon venezolanus.

\begin{tabular}{|c|c|c|c|}
\hline LOCALITY & LATITUDE & LONGITUDE & SOURCE \\
\hline Brazil, AM, PARNA Jau ${ }^{1}$ & $01^{\circ} 54^{\prime} 45^{\prime \prime} \mathrm{S}$ & $61^{\circ} 35^{\prime} 20^{\prime \prime} \mathrm{W}$ & Neckel-Oliveira and Gordo (2004) \\
\hline Colômbia, Guainia, Caño Caiman (parte alta) ${ }^{1}$ & $03^{\circ} 37^{\prime} 20^{\prime \prime} \mathrm{N}$ & $67^{\circ} 57^{\prime} 7^{\prime \prime} \mathrm{W}$ & Lynch and Ramírez (2000) \\
\hline Colômbia, Guainia, Caño Caiman (parte baja) ${ }^{1}$ & $03^{\circ} 39^{\prime} 16.5^{\prime \prime} \mathrm{N}$ & $67^{\circ} 57^{\prime} 8.5^{\prime \prime} \mathrm{W}$ & Lynch and Ramírez (2000) \\
\hline Colômbia, Guainia, La Ceiba ${ }^{1}$ & $03^{\circ} 37^{\prime} 53.2^{\prime \prime} \mathrm{N}$ & $67^{\circ} 52^{\prime} 51.1^{\prime \prime} \mathrm{W}$ & Lynch and Ramírez (2000) \\
\hline Venezuela, Amazonas, Cano Caname ${ }^{2}$ & $03^{\circ} 35^{\prime} 57.31^{\prime \prime} \mathrm{N}$ & $67^{\circ} 10^{\prime} 36.17^{\prime \prime} \mathrm{W}$ & Paolillo and Cerda (1981) \\
\hline Venezuela, Amazonas, Cano San Miguel $^{2}$ & $02^{\circ} 40^{\prime} 35.76^{\prime \prime} \mathrm{N}$ & $66^{\circ} 56^{\prime} 26.93^{\prime \prime} \mathrm{W}$ & Paolillo and Cerda (1981) \\
\hline
\end{tabular}

Coordinates: $1=$ as given in the publication; $2=$ from city town; $3=$ obtained through Google Earth software.

TABLE 2. Localities for Aparasphenodon arapapa.

\begin{tabular}{llll}
\hline LOCALITY & LATITUDE & LONGITUDE & SOURCE \\
\hline Brazil, BA, Cairu, Faz. Subaúma $^{1}$ & $13^{\circ} 31^{\prime} 0^{\prime \prime} \mathrm{S}$ & $39^{\circ} 2^{\prime} 60^{\prime \prime} \mathrm{W}$ & Pimenta et al. (2009) \\
Brazil, BA, Ituberá, APA Pratigi $^{1}$ & $13^{\circ} 43^{\prime} 60^{\prime \prime} \mathrm{S}$ & $39^{\circ} 1^{\prime} \mathbf{0}^{\prime \prime} \mathrm{W}$ & Pimenta et al. (2009) \\
Brazil, BA, Maraú $^{2}$ & $1^{\circ} 6^{\prime} 55.54^{\prime \prime} \mathrm{S}$ & $38^{\circ} 59^{\prime} 35.76^{\prime \prime} \mathrm{W}$ & Freitas et al. (2011) \\
Brazil, BA, Una, Acuípe $^{3}$ & $15^{\circ} 12^{\prime} 57.23^{\prime \prime} \mathrm{S}$ & $38^{\circ} 59^{\prime} 40.04^{\prime \prime} \mathrm{W}$ & ZUEC-AMP 16646 \\
\hline
\end{tabular}

Coordinates: 1 =as given in the publication; $2=$ from city town; $3=$ obtained through Google Earth software.

TABLE 3. Localities for Aparasphenodon bokermanni.

\begin{tabular}{llll}
\hline LOCALITY & LATITUDE & LONGITUDE & SOURCE \\
\hline Brazil, SP, Cananeia, PE Ilha do Cardoso $^{1}$ & $25^{\circ} 4^{\prime} 20.11^{\prime \prime} \mathrm{S}$ & $47^{\circ} 54^{\prime} 56.54^{\prime \prime}$ W & Pimenta et al. (2009) \\
Brazil, SC, Guaramirim $^{2}$ & $26^{\circ} 28^{\prime} 24.64^{\prime \prime} \mathrm{S}$ & $49^{\circ} 0^{\prime} 12.21^{\prime \prime}$ W & Woehl and Woehl (2003) \\
Brazil, SP, Iguape, EE Juréia Itatins $^{3}$ & $24^{\circ} 29^{\prime} 35.71^{\prime \prime} \mathrm{S}$ & $47^{\circ} 12^{\prime} 21.78^{\prime \prime}$ W & Pombal (1993) \\
Brazil, SP, Itanhaém, Cidade Santa Julia $^{3}$ & $24^{\circ} 10^{\prime} 59.07^{\prime \prime} \mathrm{S}$ & $46^{\circ} 47^{\prime} 21.75^{\prime \prime}$ W & MZUSP 56409 \\
\hline
\end{tabular}

Coordinates: 1 =as given in the publication; 2 =from city town; $3=$ obtained through Google Earth software. 
TABLE 4. Localities for Aparasphenodon brunoi.

\begin{tabular}{|c|c|c|c|}
\hline LOCALITY & LATITUDE & LONGITUDE & SOURCE \\
\hline Brazil, BA, Canavieiras, Faz. Montecristo ${ }^{2}$ & $15^{\circ} 40^{\prime} 40.44^{\prime \prime} \mathrm{S}$ & $38^{\circ} 56^{\prime} 55.54^{\prime \prime} \mathrm{W}$ & Silvano and Pimenta (2003) \\
\hline Brazil, BA, Porto Seguro, Arraial D’Ajuda ${ }^{3}$ & $16^{\circ} 29^{\prime} 23.76^{\prime \prime} \mathrm{S}$ & $39^{\circ} 4^{\prime} 58.06^{\prime \prime} \mathrm{W}$ & MBML-Anfibios 2358 \\
\hline Brazil, BA, Porto Seguro, Caraíva ${ }^{3}$ & $16^{\circ} 48^{\prime} 0^{\prime \prime} \mathrm{S}$ & $39^{\circ} 8^{\prime} 60^{\prime \prime} \mathrm{W}$ & CFBH 13285.0 \\
\hline Brazil, BA, Porto Seguro, PARNA Pau Brasil ${ }^{3}$ & $16^{\circ} 30^{\prime} 49.54^{\prime \prime} \mathrm{S}$ & $39^{\circ} 15^{\prime} 48.14^{\prime \prime} \mathrm{W}$ & Silvano and Pimenta (2003) \\
\hline Brazil, BA, Porto Seguro, RPPN Veracel ${ }^{3}$ & $16^{\circ} 23^{\prime} 13.38^{\prime \prime} \mathrm{S}$ & $39^{\circ} 8^{\prime} 45.9^{\prime \prime} \mathrm{W}$ & Pimenta et al. (2009) \\
\hline Brazil, BA, Porto Seguro, Trancoso ${ }^{1}$ & $16^{\circ} 37^{\prime} 43.21^{\prime \prime} \mathrm{S}$ & $39^{\circ} 5^{\prime} 37.29^{\prime \prime} \mathrm{W}$ & Rocha et al. (2008) \\
\hline Brazil, BA, Una, ESEX Lemos Maia ${ }^{1}$ & $15^{\circ} 18^{\prime} 0^{\prime \prime} \mathrm{S}$ & $39^{\circ} 4^{\prime} 60^{\prime \prime} \mathrm{W}$ & Argôlo (2000) \\
\hline Brazil, ES, Aracruz ${ }^{2}$ & $19^{\circ} 49^{\prime} 10.48^{\prime \prime} \mathrm{S}$ & $40^{\circ} 16^{\prime} 27.63^{\prime \prime} \mathrm{W}$ & Kasahara et al. (2003) \\
\hline Brazil, ES, Aracruz, Barra do Sahi ${ }^{3}$ & $19^{\circ} 52^{\prime} 43.14^{\prime \prime} \mathrm{S}$ & $40^{\circ} 5^{\prime} 1.97 ” \mathrm{~W}$ & MBML-Anfibios 4739 \\
\hline Brazil, ES, Aracruz, Pau Brasil ${ }^{3}$ & $19^{\circ} 51^{\prime} 0^{\prime \prime} \mathrm{S}$ & $40^{\circ} 8^{\prime} 60^{\prime \prime} \mathrm{W}$ & MBML-Anfibios 923 \\
\hline Brazil, ES, Aracruz, Santa Cruz ${ }^{3}$ & $19^{\circ} 56^{\prime} 2.61^{\prime \prime} \mathrm{S}$ & $40^{\circ} 8^{\prime} 18.89^{\prime \prime} \mathrm{W}$ & MBML-Anfibios 3 \\
\hline Brazil, ES, Aracruz, Ter. Aqu. Barra do Riacho ${ }^{3}$ & $19^{\circ} 49^{\prime} 52.83^{\prime \prime} \mathrm{S}$ & $40^{\circ} 3^{\prime} 37.19^{\prime \prime} \mathrm{W}$ & MBML-Anfibios 7202 \\
\hline Brazil, ES, Conceição da Barra, Itaúnas³ & $18^{\circ} 25^{\prime} 27.73^{\prime \prime} \mathrm{S}$ & $39^{\circ} 42^{\prime} 18.25^{\prime \prime} \mathrm{W}$ & CFBH 2394.0 \\
\hline Brazil, ES, Conceição da Barra, PE de Itaúnas ${ }^{3}$ & $18^{\circ} 24^{\prime} 33.17^{\prime \prime} \mathrm{S}$ & $39^{\circ} 42^{\prime} 54.61^{\prime \prime} \mathrm{W}$ & MBML-Anfibios 4846 \\
\hline Brazil, ES, Guarapari, PE Paulo César Vinha ${ }^{3}$ & $20^{\circ} 35^{\prime} 37.93^{\prime \prime} \mathrm{S}$ & $40^{\circ} 24^{\prime} 46.27^{\prime \prime} \mathrm{W}$ & Nunes and Fagundes (2008) \\
\hline Brazil, ES, Guarapari, Restinga de Setiba ${ }^{3}$ & $20^{\circ} 40^{\prime} 0^{\prime \prime} \mathrm{S}$ & $40^{\circ} 29^{\prime} 51^{\prime \prime} \mathrm{W}$ & MBML-Anfibios 4738 \\
\hline Brazil, ES, Ibiraçu, Faz. do Morro das Palmas-Picuãn ${ }^{3}$ & $19^{\circ} 51^{\prime} 55^{\prime \prime} \mathrm{S}$ & $40^{\circ} 20^{\prime} 10^{\prime \prime} \mathrm{W}$ & MBML-Anfibios 7419 \\
\hline Brazil, ES, Itapemirim, Lagoa Sete Pontas ${ }^{3}$ & $20^{\circ} 55^{\prime} 59.48^{\prime \prime} \mathrm{S}$ & $40^{\circ} 49^{\prime} 29.39^{\prime \prime} \mathrm{W}$ & MBML-Anfibios 4743 \\
\hline Brazil, ES, Linhares, Estrada Linhares-Povoação³ & $19^{\circ} 28^{\prime} 30.83^{\prime \prime} \mathrm{S}$ & $39^{\circ} 54^{\prime} 8.92^{\prime \prime} \mathrm{W}$ & MBML-Anfibios 2018 \\
\hline Brazil, ES, Linhares, Paraju RFCVRD ${ }^{3}$ & $19^{\circ} 6^{\prime} 44.73^{\prime \prime} \mathrm{S}$ & $39^{\circ} 57^{\prime} 38.75^{\prime \prime} \mathrm{W}$ & ZUEC-AMP 9769 \\
\hline Brazil, ES, Praia das Neves ${ }^{1}$ & $21^{\circ} 15^{\prime} 0^{\prime \prime} \mathrm{S}$ & $40^{\circ} 58^{\prime} 0^{\prime \prime} \mathrm{W}$ & Rocha et al. (2008) \\
\hline Brazil, ES, Presidente Kennedy ${ }^{1}$ & $21^{\circ} 17^{\prime} 59^{\prime \prime} \mathrm{S}$ & $40^{\circ} 57^{\prime} 30^{\prime \prime} \mathrm{W}$ & Wogel et al. (2006) \\
\hline Brazil, ES, Presidente Kennedy ${ }^{1}$ & $21^{\circ} 17^{\prime} 40^{\prime \prime} \mathrm{S}$ & $40^{\circ} 57^{\prime} 34.99^{\prime \prime} \mathrm{W}$ & Wogel et al. (2006) \\
\hline Brazil, ES, Presidente Kennedy, Praia das Neves ${ }^{4}$ & $21^{\circ} 16^{\prime} 48^{\prime \prime} \mathrm{S}$ & $40^{\circ} 58^{\prime} 9^{\prime \prime} \mathrm{W}$ & MBML-Anfibios 4672 \\
\hline Brazil, ES, São Mateus, APA de Restinga de Guriri ${ }^{3}$ & $18^{\circ} 49^{\prime} 33.91^{\prime \prime} \mathrm{S}$ & $39^{\circ} 45^{\prime} 32.9^{\prime \prime} \mathrm{W}$ & MBML-Anfibios 1803 \\
\hline Brazil, ES, São Mateus, Fazenda Lagoa Suruaca ${ }^{4}$ & $19^{\circ} 8^{\prime} 18^{\prime \prime} \mathrm{S}$ & $39^{\circ} 43^{\prime} 56^{\prime \prime} \mathrm{W}$ & MBML-Anfibios 2377 \\
\hline Brazil, ES, São Mateus, São Mateus² & $18^{\circ} 42^{\prime} 58^{\prime \prime} \mathrm{S}$ & $39^{\circ} 51^{\prime} 32^{\prime \prime} \mathrm{W}$ & MBML-Anfibios 695 \\
\hline Brazil, ES, Setiba $^{1}$ & $20^{\circ} 34^{\prime} 60^{\prime \prime} \mathrm{S}$ & $40^{\circ} 27^{\prime} 0^{\prime \prime} \mathrm{W}$ & Rocha et al. (2008) \\
\hline Brazil, ES, Serra, Balneário Costa Bela ${ }^{3}$ & $20^{\circ} 10^{\prime} 22.17^{\prime \prime} \mathrm{S}$ & $40^{\circ} 11^{\prime} 14.09^{\prime \prime} \mathrm{W}$ & MBML-Anfibios 7386 \\
\hline Brazil, ES, Sooretama ${ }^{2}$ & $19^{\circ} 11^{\prime} 50.27^{\prime \prime} \mathrm{S}$ & $40^{\circ} 5^{\prime} 52.74^{\prime \prime} \mathrm{W}$ & MZUSP 124721-22, MZUSP 125027 \\
\hline Brazil, ES, Vila Velha, Lagoa do Milho, Ponta da Fruta ${ }^{2}$ & $20^{\circ} 19^{\prime} 47^{\prime \prime} \mathrm{S}$ & $40^{\circ} 17^{\prime} 32^{\prime \prime} \mathrm{W}$ & ZUEC-AMP 3725 \\
\hline Brazil, ES, Vitória, Goiabeiras ${ }^{3}$ & $20^{\circ} 15^{\prime} 33.87^{\prime \prime} \mathrm{S}$ & $40^{\circ} 17^{\prime} 20.75^{\prime \prime} \mathrm{W}$ & MBML-Anfibios 2109 \\
\hline Brazil, ES, Vitória, Restinga de Camburi ${ }^{3}$ & $20^{\circ} 15^{\prime} 58.18^{\prime \prime} \mathrm{S}$ & $40^{\circ} 15^{\prime} 55.31^{\prime \prime} \mathrm{W}$ & MBML-Anfibios 4746 \\
\hline Brazil, MG, Marliéria, PE Rio do Doce ${ }^{1}$ & $19^{\circ} 41^{\prime} 58.67^{\prime \prime} \mathrm{S}$ & $42^{\circ} 31^{\prime} 26.12^{\prime \prime} \mathrm{W}$ & Feio et al. (1999) \\
\hline Brazil, RJ, Maricá ${ }^{1}$ & $22^{\circ} 57^{\prime} 18^{\prime \prime} \mathrm{S}$ & $42^{\circ} 49^{\prime} 48.72^{\prime \prime} \mathrm{W}$ & Rocha et al. (2008) \\
\hline Brazil, RJ, Guapimirim, RPPN Campo Esc. G.H. Nunes ${ }^{3}$ & $22^{\circ} 34^{\prime} 47.24^{\prime \prime} \mathrm{S}$ & $43^{\circ} 2^{\prime} 1.64^{\prime \prime} \mathrm{W}$ & Silva-Soares et al. (2010) \\
\hline Brazil, RJ, Grumari ${ }^{1}$ & $23^{\circ} 3^{\prime} 0^{\prime \prime} \mathrm{S}$ & $43^{\circ} 31^{\prime} 60^{\prime \prime} \mathrm{W}$ & Rocha et al. (2008) \\
\hline Brazil, RJ, Jurubatiba ${ }^{1}$ & $22^{\circ} 16^{\prime} 60^{\prime \prime} \mathrm{S}$ & $41^{\circ} 40^{\prime} 59.99^{\prime \prime} \mathrm{W}$ & Rocha et al. (2008) \\
\hline Brazil, RJ, Massambaba ${ }^{1}$ & $22^{\circ} 55^{\prime} 60^{\prime \prime} \mathrm{S}$ & $42^{\circ} 12^{\prime} 0^{\prime \prime} \mathrm{W}$ & Rocha et al. (2008) \\
\hline Brazil, RJ, Rio de Janeiro, Barra da Tijuca ${ }^{3}$ & $22^{\circ} 56^{\prime} 35.7^{\prime \prime} \mathrm{S}$ & $43^{\circ} 17^{\prime} 19.29^{\prime \prime} \mathrm{W}$ & Pimenta et al. (2009) \\
\hline Brazil, RJ, Rio de Janeiro, Recreio dos Bandeirantes ${ }^{3}$ & $23^{\circ} 1^{\prime} 10.49^{\prime \prime} \mathrm{S}$ & $43^{\circ} 28^{\prime} 45.24^{\prime \prime} \mathrm{W}$ & ZUEC-AMP 1969 \\
\hline Brazil, RJ, Rio de Janeiro, Restinga de Grumari ${ }^{3}$ & $23^{\circ} 2^{\prime} 42.06^{\prime \prime} \mathrm{S}$ & $43^{\circ} 32^{\prime} 3.31^{\prime \prime} \mathrm{W}$ & ZUEC-AMP 2758 \\
\hline Brazil, RJ, Casemiro de Abreu, Barra do São João ${ }^{3}$ & $22^{\circ} 35^{\prime} 32.3^{\prime \prime} \mathrm{S}$ & $41^{\circ} 59^{\prime} 26.53^{\prime \prime} \mathrm{W}$ & MZUSP 123705, MZUSP 123714-15 \\
\hline Brazil, SP, Caraguatatuba $^{5}$ & $23^{\circ} 39^{\prime} 26.6^{\prime \prime} \mathrm{S}$ & $45^{\circ} 25^{\prime} 56.62^{\prime \prime} \mathrm{W}$ & New Record (MZUSP 149809) \\
\hline Brazil, SP, Ubatuba, Praia do Lázaro ${ }^{3}$ & $23^{\circ} 30^{\prime} 3.01^{\prime \prime} \mathrm{S}$ & $45^{\circ} 8^{\prime} 19.55^{\prime \prime} \mathrm{W}$ & $\begin{array}{l}\text { Sazima and Cardoso (1980); Gioia and Souza- } \\
\text { Lima (1988) }\end{array}$ \\
\hline
\end{tabular}

Coordinates: $1=$ given in the publication; $2=$ from city town; $3=$ obtained through Google Earth software; $4=$ given on SpeciesLink web portal; 5=took with a GPS.

Aparasphenodon brunoi is currently considered of "least concern" by the IUCN because it is widely distributed (Rocha et al. 2004). However the populations are decreasing, probably due to habitat loss as we observed in Caraguatatuba, and due to other human activities along the Brazilian sandy coastal plains, like human settlements (Rocha et al. 2004).

Thus the new record presented here is of interest to the understanding of how climatic changes can affect species range expansion, a relatively well documented phenomenon for species inhabiting temperate areas (Melles et al. 2011; Imbert et al. 2012; Mair et al. 2012), but that is still poorly addressed in tropical environments, such as the Atlantic Forest. Our results also point out that after more than 20 years without any further record Aparasphenodon brunoi is still present in the restinga forests of São Paulo state, a very anthropized environment in the São Paulo's Atlantic Forest, which obviously needs urgent conservation plans. 

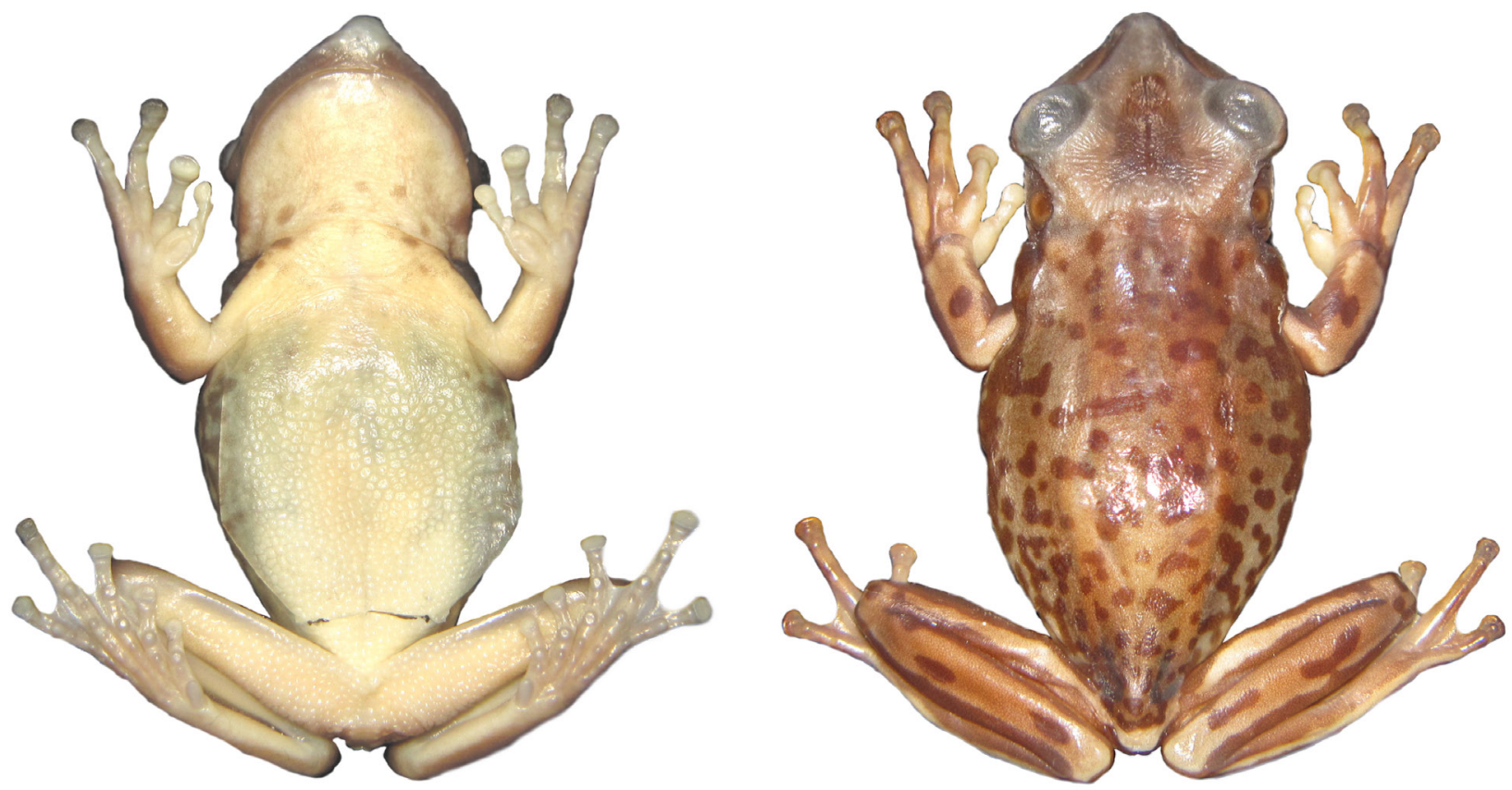

FIGURE 2. Specimen of Aparasphenodon brunoi (MZUSP 149809) collected in Caraguatatuba, São Paulo, Brazil.

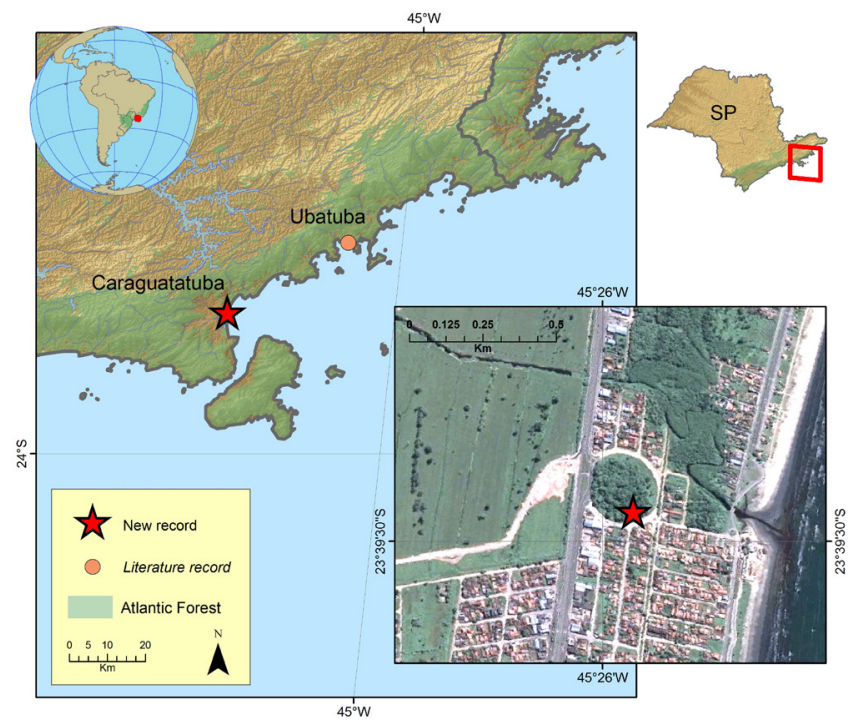

FIGURE 3. New record of Aparasphenodon brunoi in São Paulo state, Brazil: Caraguatatuba, on the coastal plain, and the only other known record for São Paulo state, at Ubatuba, Lázaro beach (Gioia and SouzaLima 1988). SP=São Paulo state.

ACKNowhedgments: We are grateful to Hussam Zaher and Carolina Castro Mello for allowing access to specimens housed in the Museu de Zoologia da Universidade de São Paulo. We also would like to thank Maria Strangas for reviewing the English and Carla Piantoni for comments and suggestions on early version of this manuscript. We also thank Philippe Kok and an anonymous reviewer for comments and suggestions on this manuscript.

\section{LITERATURE CiTED}

Argolo, A.J.S. 2000. Aparasphenodon brunoi. Herpetological Review 31(2): 108.

Carvalho, A.L. 1941. Notas sôbre os gêneros Corythomantis Boulenger e Aparasphenodon Miranda Ribeiro (Amphibia-Anura, Hylidae). Papéis Avulsos do Departamento de Zoologia 1(14): 101-100.

Feio, R.N., P.S. Santos and U. Caramaschi. 1999. New Records of Amphibians from Parque Estadual do Rio Doce, State of Minas Gerais, Brazil. Herpetological Review 30(1): 57.

Freitas, M.A. 2011. Anfibios do Nordeste Brasileiro. Mata Atlântica - Caatinga - Cerrado - Zona Costeira - Amazônia. (produced by the author): (produced by the author). $86 \mathrm{p}$.
GBIF. 2011. Global Biodiversity Information Facility - Data Portal.Global Biodiversity Information Facility. Accessible at http://data.gbif.org/. Captured on 6 January 2012.

Gioia, I. and R. Souza Lima. 1988. Zelleriella bromelicola sp. n. (Protozoa: Opalinatea): entozoario de Aparasphenodon brunoi MirandaRibeiro, 1920 (Anura, Hylidae) de Ubatuba, Sao Paulo, Brasil. Revista Brasileira de Zoologia 5(2): 215-220.

Imbert, C.E., F. Goussard and A. Roques. 2012. Is the expansion of the pine processionary moth, due to global warming, impacting the endangered Spanish moon moth through an induced change in food quality? Integrative Zoology 7(2): 147-157.

Kasahara, S., A.P.Z. Silva, S.L. Gruber and C.F.B. Haddad. 2003. Comparative cytogenetic analysis on four tree frog species (Anura, Hylidae, Hylinae) from Brazil. Cytogenetic and Genome Research 103(1-2): 155-162.

Lutz, A. 1925. Batraciens du Brésil. Comptes Rendus et Mémoires Hebdomadaires des Séances de la Société de Biologie et des ses Filiales 93(21): 137-139.

Lynch, J.D. and M.A.V. Ramírez. 2000. Lista Preliminar de Especies de Anuras del Departamento del Guainia, Colombia. Revista de la Academia Colombiana de Ciencias Exactas, Físicas y Naturales 24(93): 579-589.

Mair, L., C.D. Thomas, B.J. Anderson, R. Fox, M. Botham and J.K. Hill. 2012. Temporal variation in responses of species to four decades of climate warming. Global Change Biology 18(8): 2439-2447.

Melles, S.J., M.J. Fortin, K. Lindsay and D. Badzinski. 2011. Expanding northward: influence of climate change, forest connectivity, and population processes on a threatened species' range shift. Global Change Biology 17(1): 17-31.

Mertens, R. 1950. Ein neuer Laubfrosch aus Venezuela. Senckenbergiana Biologica 31: 1-10.

Mesquita, D.O., G.C. Costa and M.G. Zatz. 2004. Ecological aspects of the casque-headed frog Aparasphenodon brunoi (Anura, Hylidae) in a Restinga habitat in southeastern Brazil. Phyllomedusa 3(1): 51-60.

Miranda-Ribeiro, A. 1920. Triprion, Diaglena, Corythomantis, etc. uma subsecção de Hylidae, com duas especies novas. Revista do Museu Paulista 12: 85-89.

Miranda-Ribeiro, P. 1955. Tipos das espécies e subespécies do Prof Alipioi de Miranda Ribeiro depositados no Museu Nacional. Arquivos do Museu Nacional 42: 389-417.

Neckel-Oliveira, S. and M. Gordo. 2004. Aparasphenodon venezolanus (Venezuela casque-headed frog). Herpetological Review 35(1): 77.

Nunes, R.R.A. and V. Fagundes. 2008. Karyotypes of amphibians of the subfamilies Hylinae and Phyllomedusinae (Anura, Hylidae) from Espirito Santo, Brazil. Boletim do Museu de Biologia Mello Leitao 23: 21-36.

Paolillo, A. and J. Cerda. 1981. Nuevos hallazgos de Aparasphenodon venezolanus (Mertens) (Salientia, Hylidae) en el Territorio Federal Amazonas Venezuela, con anotaciones sobre su biologia. Memoria de la Sociedad de Ciencias Naturales La Salle 41: 77-95. 
Pimenta, B.V.S., M.F. Napoli and C.F.B. Haddad. 2009. A new species of casque-headed tree frog, genus Aparasphenodon Miranda-Ribeiro (Amphibia: Anura: Hylidae), from the Atlantic Rainforest of southern Bahia, Brazil. Zootaxa 2009(2123): 46-54.

Pombal, J.P., Jr. 1993. New species of Aparasphenodon (Anura: Hylidae) from southeastern Brazil. Copeia 4: 1088-1091.

Rocha, C.F.D., S.P. Carvalho e Silva and M. Van Sluys. 2004. Aparasphenodon brunoi. IUCN 2011. IUCN Red List of Threatened Species. Version 2011.2. Accessible at www.iucnredlist.org. Captured on 09 June 2012.

Rocha, C.F.D., F.H. Hatano, D. Vrcibradic and M. Van Sluys. 2008. Frog species richness, composition and beta-diversity in coastal Brazilian restinga habitats. Brazilian Journal of Biology 68(1): 101-107.

Sazima, I. and A.J. Cardoso. 1980. Notas sobre a distribuição de Corythomantis greeningi Boulenger, 1896 e Aparasphenodon brunoi Miranda-Ribeiro, 1920 (Amphibia, Hylidae). Iheringia Serie Zoologia 55: 3-7.

Sena, M.A., J. Cassimiro, C.J. David, J.G. Da Silva and M.T. Rodrigues. 2008. Tropidurus torquatus (calango, collared lizard). Herpetological Review 39(3): 369.

Silva-Soares, T., F. Hepp, P.N. Costa, C. de Luna-Dias, M.d.R. Gomes, S.P. Carvalho e Silva and A.M.P.T. de Carvalho e Silva. 2010. Anuran Amphibians from RPPN Campo Escoteiro Geraldo Hugo Nunes, Guapimirim Municipality, Rio de Janeiro, Southeastern Brazil. Biota Neotropica 10(2): 225-233.

Silvano, D.L. and B.V.S. Pimenta. 2003. Diversidade e Distribuição de Anfíbios na Mata Atlântica do Sul da Bahia; p. 1-22 In P.I. Prado, E.C. Landau, R.T. Moura, L.P.S. Pinto, G.A.B. Fonseca and K. Alger (ed.). Corredor de Biodiversidade na Mata Atlântica do Sul da Bahia. Ilhéus,BA: ISEB/CI/CABS/UFMG/UNICAMP.
SpeciesLink. 2011. Rede SpeciesLink.Centro de Referência em Informação Ambiental (CRIA), Fundação de Amparo à Pesquisa do Estado de São Paulo (FAPESP). Accessible at http://www.splink.org.br/. Captured on 6 January 2012.

Teixeira, R.L., J.A.P. Schineider and G.I. Almeida. 2002. The occurrence of amphibians in bromeliads from a southeastern Brazilian restinga habitat, with special reference to Aparasphenodon brunoi (Anura, Hylidae). Brazilian Journal of Biology 62(2): 263-268.

Trueb, L. 1970. Evolutionary relationships of casque-headed tree frog with co-ossified skulls (family Hylidae). University of Kansas Publications of the Museum of Natural History 18: 547-716

Woehl, G., Jr. and E.N. Woehl. 2003. Aparasphenodon bokermanni. Herpetological Review 34(4): 379.

Wogel, H., L.N. Weber and P.A. Abrunhosa. 2006. The tadpole of the casque-headed frog, Aparasphenodon brunoi Miranda-Ribeiro (Anura: Hylidae). South American Journal of Herpetology 1(1): 54-60.

RECEIVED: July 2012

ACCEPTED: October 2012

Published ONLINE: December 2012

EDITORIAL RESPONSIBILITY: Philippe J.R. Kok 